\title{
Identifying barriers for out of hospital emergency care in low and low-middle income countries: a systematic review
}

Antony Gatebe Kironji ${ }^{*}$, Peter Hodkinson², Sarah Stewart de Ramirez ${ }^{3}$, Trisha Anest ${ }^{3}$, Lee Wallis², Junaid Razzak Alexander Jenson ${ }^{3}$ and Bhakti Hansoti ${ }^{2,3}$

\begin{abstract}
Background: Out-of-hospital emergency care (OHEC), also known as prehospital care, has been shown to reduce morbidity and mortality from serious illness. We sought to summarize literature for low and low-middle income countries to identify barriers to and key interventions for OHEC delivery.

Methods: We performed a systematic review of the peer reviewed literature from January 2005 to March 2015 in PubMed, Embase, Cochrane, and Web of Science. All articles referencing research from low and low-middle income countries addressing OHEC, emergency medical services, or transport/transfer of patients were included. We identified themes in the literature to form six categories of OHEC barriers. Data were collected using an electronic form and results were aggregated to produce a descriptive summary.

Results: A total 1927 titles were identified, 31 of which met inclusion criteria. Barriers to OHEC were divided into six categories that included: culture/community, infrastructure, communication/coordination, transport, equipment and personnel. Lack of transportation was a common problem, with 55\% (17/31) of articles reporting this as a hindrance to OHEC. Ambulances were the most commonly mentioned $(71 \%, 22 / 31)$ mode of transporting patients. However, many patients still relied on alternative means of transportation such as hired cars, and animal drawn carts. Sixty-one percent (19/31) of articles identified a lack of skilled personnel as a key barrier, with 32\% (10/31) of OHEC being delivered by laypersons without formal training. Forty percent (12/31) of the systems identified in the review described a uniform access phone number for emergency medical service activation.
\end{abstract}

Conclusions: Policy makers and researchers seeking to improve OHEC in low and low-middle income countries should focus on increasing the availability of transport and trained providers while improving patient access to the OHEC system. The review yielded articles with a primary focus in Africa, highlighting a need for future research in diverse geographic areas.

Keywords: Emergency medicine, Barriers to prehospital care, Prehospital care, Out of hospital emergency care, Low-middle income countries (LMIC), Low income countries (LIC)

\footnotetext{
* Correspondence: Kironji@jhmi.edu

'Johns Hopkins School of Medicine, Baltimore, MD, USA

Full list of author information is available at the end of the article
}

(c) The Author(s). 2018 Open Access This article is distributed under the terms of the Creative Commons Attribution 4.0 International License (http://creativecommons.org/licenses/by/4.0/), which permits unrestricted use, distribution, and reproduction in any medium, provided you give appropriate credit to the original author(s) and the source, provide a link to the Creative Commons license, and indicate if changes were made. The Creative Commons Public Domain Dedication waiver (http://creativecommons.org/publicdomain/zero/1.0/) applies to the data made available in this article, unless otherwise stated. 


\section{Background}

The development of emergency care systems is a growing focus in low-middle income countries (LMIC). It is estimated that as many as $45 \%$ of deaths and $35 \%$ of disability-adjusted life years can be addressed by developing robust emergency care systems in LMIC [1]. One of the biggest challenges in many rural low resource settings is the scarcity of emergency care, and where present, the distance and time to access appropriate services [2-4].

Out of Hospital Emergency Care (OHEC), commonly understood as prehospital care, refers to the acute and emergency care delivered outside the walls of a fixed health facility/hospital [5]. It has previously been demonstrated that in LMIC without formal emergency care systems, nearly $80 \%$ of deaths due to severe injury occurred in the prehospital setting [6]. Developing prehospital trauma care systems has been emphasized as an integral component of the healthcare system [7].

OHEC includes a spectrum of care delivery from first responder care (FRC), prehospital care (PHC), and emergency medical services (EMS). OHEC is an umbrella term coined by the African Federation for Emergency Medicine (AFEM) in 2013. It begins with first responder care upon recognition of a perceived or actual emergency and includes the full spectrum of emergency care that occurs outside of healthcare facilities.

Less than $1 \%$ of the population in many lowincome countries (LIC) has access to formal emergency medical transportation services, such as ambulances [8]. In sub-Saharan Africa and Asia those few who do have access to medical transport would typically only have transport between facilities and not from the scene of injury [9]. Delays in reaching health facilities has been shown to lead to poor patient outcomes [3], yet up to $60 \%$ of individuals living in a LMIC live more than $8 \mathrm{~km}$ away from the nearest health facility/hospital [10]. Given the scarcity of emergency care facilities in LMIC, [5] OHEC can be crucial in determining patient survival.

The majority of the literature to date in LMIC focuses on barriers for OHEC in specific countries often with disease specific focuses (e.g. trauma or obstetric care) [11-18]. Additionally, the most recent review that describes barriers to emergency care was conducted in 2012 [19]. While this study focuses on barriers to care in both high and low income countries, that study predominantly emphasized financial barriers to care delivery. This review performs a comprehensive systematic search of the peer-reviewed literature across four different databases to identify barriers to and also key interventions for OHEC delivery in LMIC.

\section{Methods}

\section{Criteria for considering studies in this review}

This systematic review included review articles, observational studies, quantitative studies, qualitative studies, evaluations/report articles and policy papers that addressed OHEC. This included articles that addressed (1) emergency care for patients prior to arriving at the hospital (first responder care or prehospital care) and (2) systems in place to respond to health emergencies. All health conditions were included. Studies that did not include LIC or LMIC as defined by the World Bank (http://data. worldbank.org/about/country-and-lending-groups), studies focusing only on care in hospital settings, case based studies $(n<5)$, editorials or descriptive studies were excluded (Table 1).

\section{Search methods for identification of studies}

We searched MEDLINE (via PubMed), Embase, Cochrane, and Web of Science to find potential articles from January 2005 to March 2015. We included combined controlled vocabulary words $(\mathrm{MeSH})$ and related keywords for 1) barriers to care, 2) emergency medical care (FRC, PHC, and EMS) and 3) developing country. See Appendix 1: Search Strategy. This search explored database Boolean operators ('OR' for related/similar terms and 'AND' to combine different concepts) to combine these three key ideas. We consulted an informationist (KL) from Johns Hopkins University Welch Medical Library for each search strategy. Results in languages other than English were excluded.

\section{Data collection and analysis \\ Selection of studies}

We imported all results from the four data bases into Refworks for initial organization of the results. Prior to review, duplicate records were removed using Refworks. For the review, all relevant articles were exported from Refworks into Excel. A team of six researchers (GK, BH, $\mathrm{AJ}, \mathrm{PH}, \mathrm{TA}$ and SSR) were responsible for conducting

Table 1 Inclusion and exclusion criteria used to screen articles

\begin{tabular}{|c|c|}
\hline Inclusion & Exclusion \\
\hline $\begin{array}{l}\text { LIC or LMIC as defined by the } \\
\text { World Bank } \\
\text { (http://data.worldbank.org/ } \\
\text { about/country-and-lending-groups). }\end{array}$ & $\begin{array}{l}\text { Non-English language; Full article } \\
\text { not found }\end{array}$ \\
\hline $\begin{array}{l}\text { Study focus: All studies addressing } \\
\text { Out of hospital emergency care } \\
\text { which includes First responder care, } \\
\text { Prehospital care, and Emergency } \\
\text { medical care }\end{array}$ & Study focus: Hospital care \\
\hline \multicolumn{2}{|l|}{ Disease focus: All disease conditions } \\
\hline $\begin{array}{l}\text { Type of study: Observational, } \\
\text { Evaluation/reports, Expert review, } \\
\text { and Qualitative studies }\end{array}$ & $\begin{array}{l}\text { Type of study: Case based studies } \\
(n<5) \text {, editorials and descriptive } \\
\text { studies }\end{array}$ \\
\hline
\end{tabular}


the entire review process. Two reviewers independently screened each title and abstract. Using the inclusion and exclusion criteria, the reviewers evaluated the titles and abstracts to see if they should receive a full text review marking them as "Yes" or "No". Articles were marked as "No" only if they violated any of the exclusion criteria. A "Yes" vote was assigned to articles that did not violate the exclusion criteria. All articles marked "Yes" during the title and abstract review were included for the full text review. Any conflict regarding study inclusion were resolved by a third reviewer.

In the title and abstract screening process articles were excluded if they (1) didn't include at least one LIC or LMIC (2) didn't address OHEC (3) only included inhospital emergency care $(4)$ were case based studies $(n<5)$, editorials or descriptive studies (4) none English.

For the full text review, one reviewer was assigned to each article. All articles were obtained by the study team by searching google, MEDLINE, and the Johns Hopkins Welch Library online resources. The reviewers screened the full text for presences of inclusion criteria and violation of exclusion criteria. The same inclusion and exclusion criteria listed above were applied to the full-text review. After screening the text, reviewers selected to either include or exclude the article into the systematic review. The screening process was conducted in accordance with Preferred Reporting Items for Systematic Reviews and Meta-Analyses (PRISMA) guidelines, Fig. 1 shows the PRISMA process flow diagram.

\section{Data extraction and management}

Data extraction for the full text was performed by five reviewers (GK, BH, AJ, $\mathrm{PH}$, and $\mathrm{TA})$. Each article was assigned to one reviewer. All reviewers used standardized definitions and examples for the extraction process. Data was collected with an electronic form that utilized

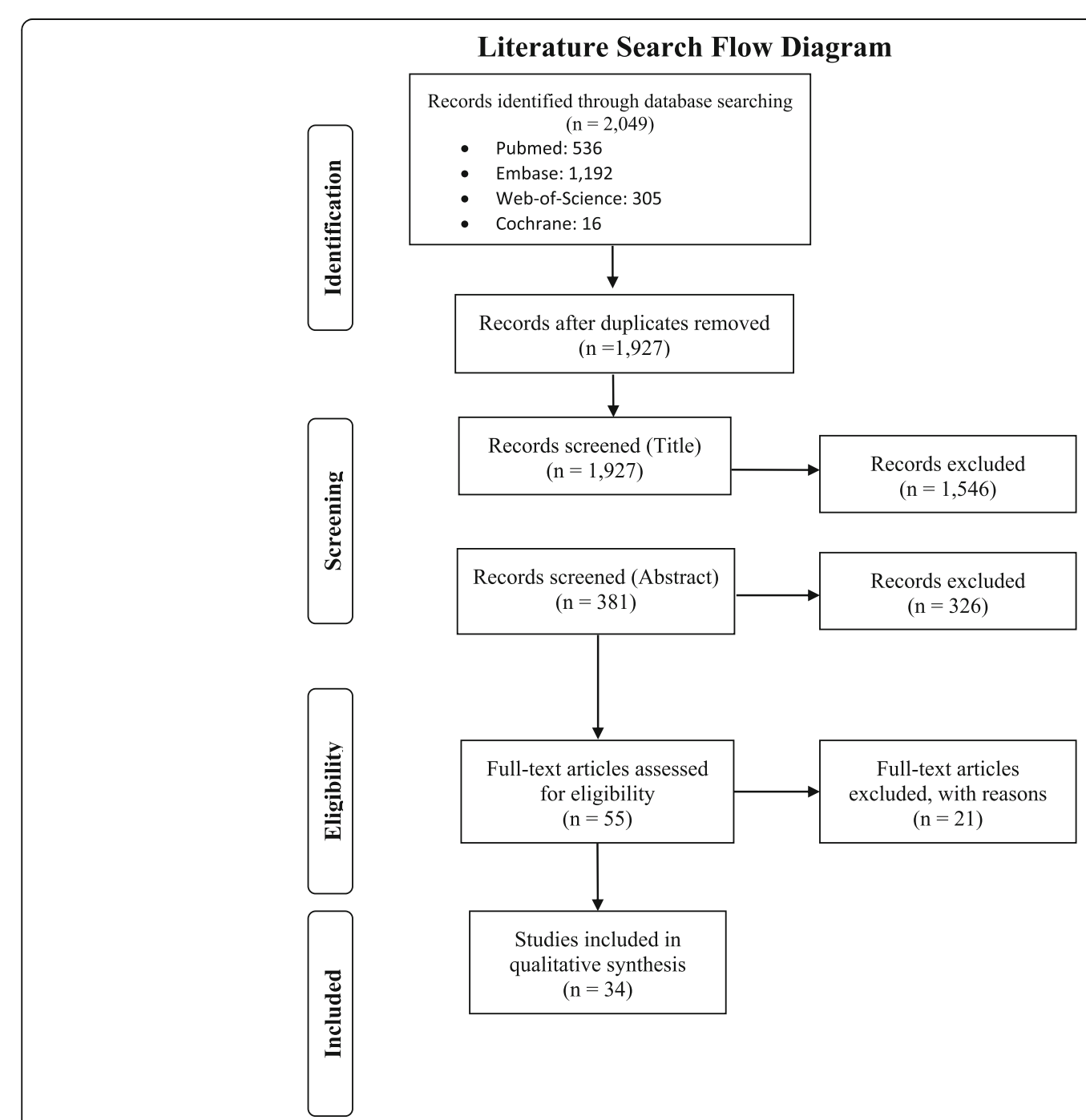

Fig. 1 Summary of sources contributing to the systematic review 
multiple choice as well as free text options to capture responses. For each article the review recorded: 1) the study site, 2) information on the OHEC system as described by the article (which included level of first responder training, types of emergency transport available, patient population being served, type of EMS system available, and availability of an emergency contact number), 3) summary of barriers to delivery of OHEC addressed in the article within the following domains: health access, communication, medical equipment, availability and training of first responders, coordination of the EMS system, triage of patients and finances, 4) summary of the EMS pathway where barriers exist (community, dispatch, emergency responders, transportation, and/or roads). See Table 2. For each barrier domain and EMS pathway selected the reviewer provided evidence from the article using quotes or paraphrasing.

\section{Synthesis of the barriers}

The barrier domains and the EMS pathway barriers were defined a priori $[9,19]$. After the data was collected reviewers combined and reorganized these two concepts into the following themes: culture/community, infrastructure, communication/coordination, transport, equipment, and personnel. Like terms and concepts were combined. This new framework simplifies how barriers are viewed, see Fig. 2.

Culture/community represents the knowledge that an individual and/or community may have to recognize an emergent health condition or cultural beliefs that affect the decision to seek urgent medical attention. Infrastructure combines concepts from transportation infrastructure (i.e. road quality, accessible roads and road networks, navigation friendly, etc.) with access to appropriate health facilities (i.e. access to community health center vs. trauma center after a major accident). Communication/coordination represents the ability for a care seeker to call for help through a designated phone number and also for the care provider to be able to coordinate the response effort: this involves effective communication between the responders, the dispatcher, and the hospital. This category also includes the ability for the dispatcher to triage patients appropriately. Transport represents the means that is used to get the patient to an appropriate health facility. Equipment represents the resuscitative equipment and medications necessary to stabilize a patient for transport to a health facility. Lastly, personnel involve the presence and training of personnel responding to emergencies.

\section{Assessing risk of bias}

Three reviewers (AJ, GK, and PH) assessed study bias using the modified RTI risk of bias tool for observational studies. Articles were assessed using the following domains: inclusion/exclusion criteria, participant recruitment, blinding of study assessors, validity/reliability of study measures, length of study follow-up, loss to follow-up, assessments of harms in study, study limitations and confounding [20]. Each reviewer was assigned a set of articles to review and results were collected using a common abstraction tool.

\section{Results \\ Overview}

Of the 1927 unique titles identified in our search strategy, 31 articles were included for full review (Table 2). Most, 77\% (24/31), identified three or more categories of barriers present within their respective health systems (Table 3). Primary authors of most of the articles, 55\% $(17 / 31)$, were from outside of the study country (Table 4). Financial constraints at the level of the care-seeker, the institution and the country had significant impacts at multiple levels in the development of OHEC and were not further explored in this review.

\section{Culture/community}

Several studies focused on differences in culture and impact on healthcare seeking behavior. For example, in the obstetric literature, it has been reported that once a patient recognizes a need for medical attention, the decision to seek care is not solely their own; decisions to seek care may be influenced by the mother-in-law $[3,21]$ husband/male relatives $[8,21]$, or a village elders [11]. For some, more familiar traditional approaches were preferred to newer methods that had not yet gained widespread community approval $[12,14,18,21]$. Knowledge about and the ability to recognize medical danger signs was another area of focus for many articles [3, 11, 12, 14, 16, 21]. Authors noted that patients were often not aware of when they should seek immediate care.

\section{Infrastructure}

Poor road conditions and poor road networks were the most commonly addressed deficits, 48\% (15/31), for transportation infrastructure. Other issues that contribute to this barrier include: sharing roads between the public and emergency vehicles, increased travel distance, lack of road signs, eroded terrains, and narrow roads [2, 8, 11-13, 16, 21-23]. Seasonal difficulties, such as difficulty passing through roads during the rainy season, were commonly reported $[3,8,12,13$, 16]. In addition to poor infrastructure preventing timely access to care, some articles noted that it also contributed to increased frequency of traffic accidents [2, 22, 24] and increased concerns for transporting patients safely to health facilities $[2,3]$. Of the articles that identified an infrastructure barrier, 59\% (10/17) noted that patients were 


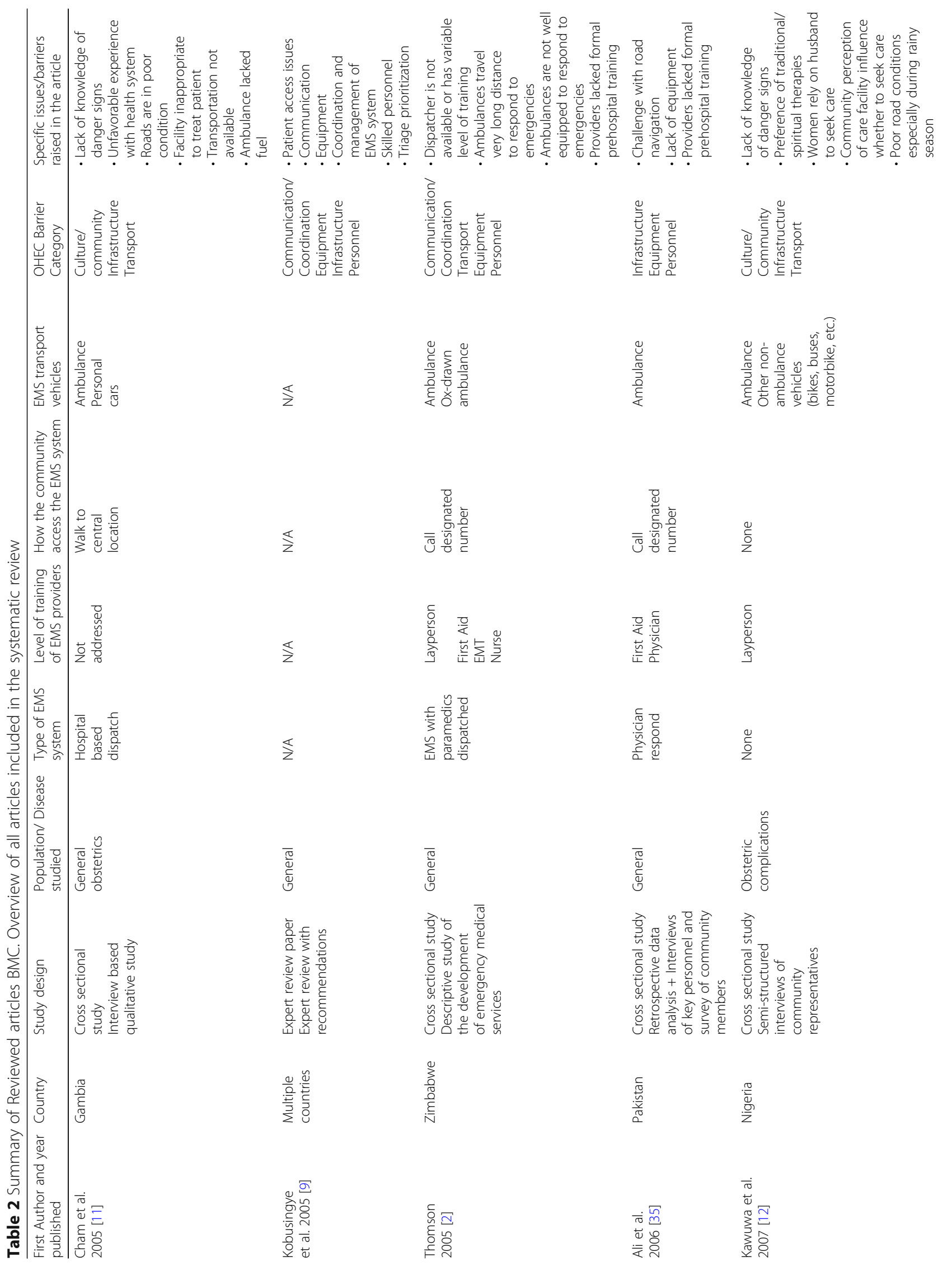




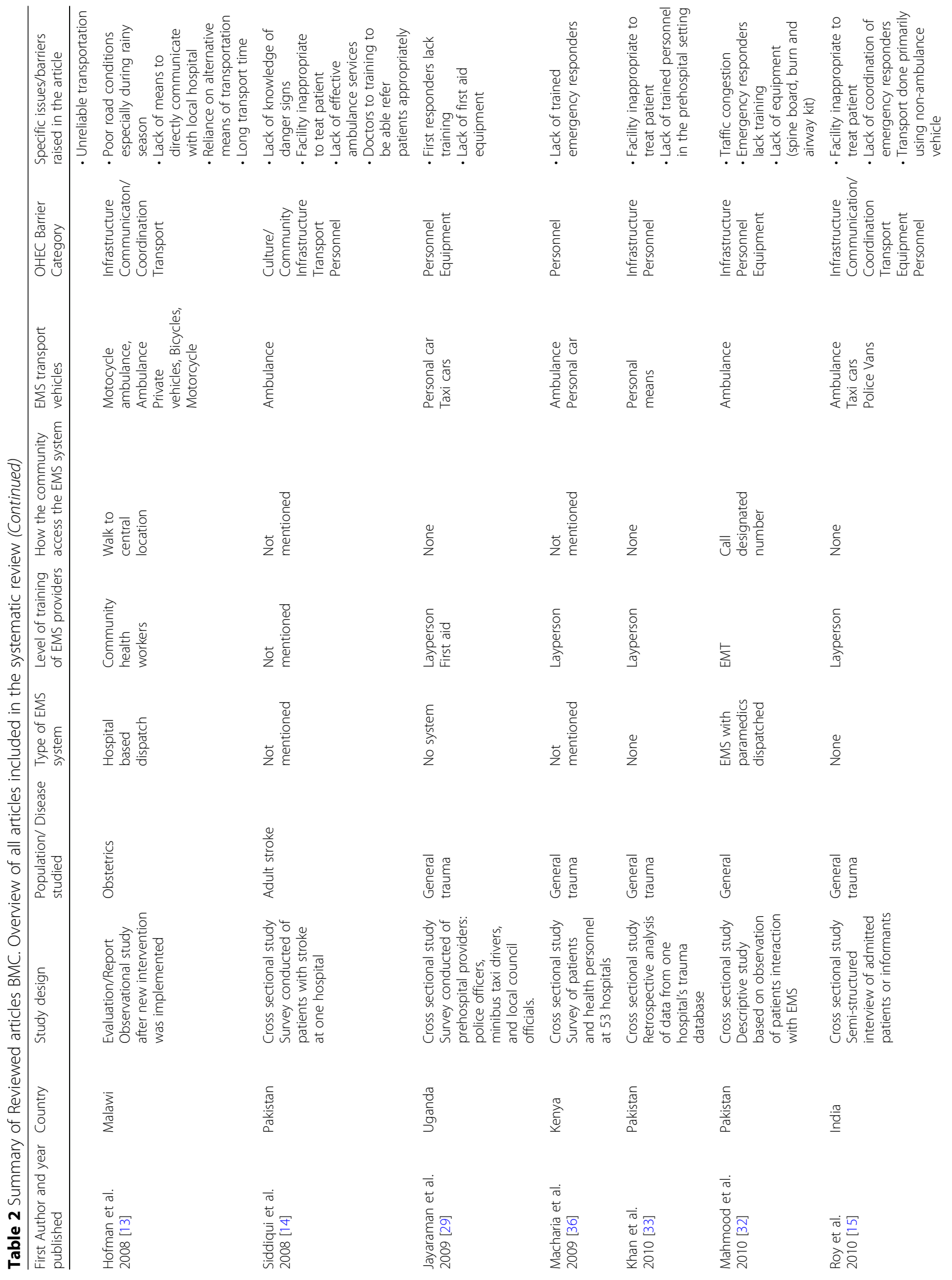




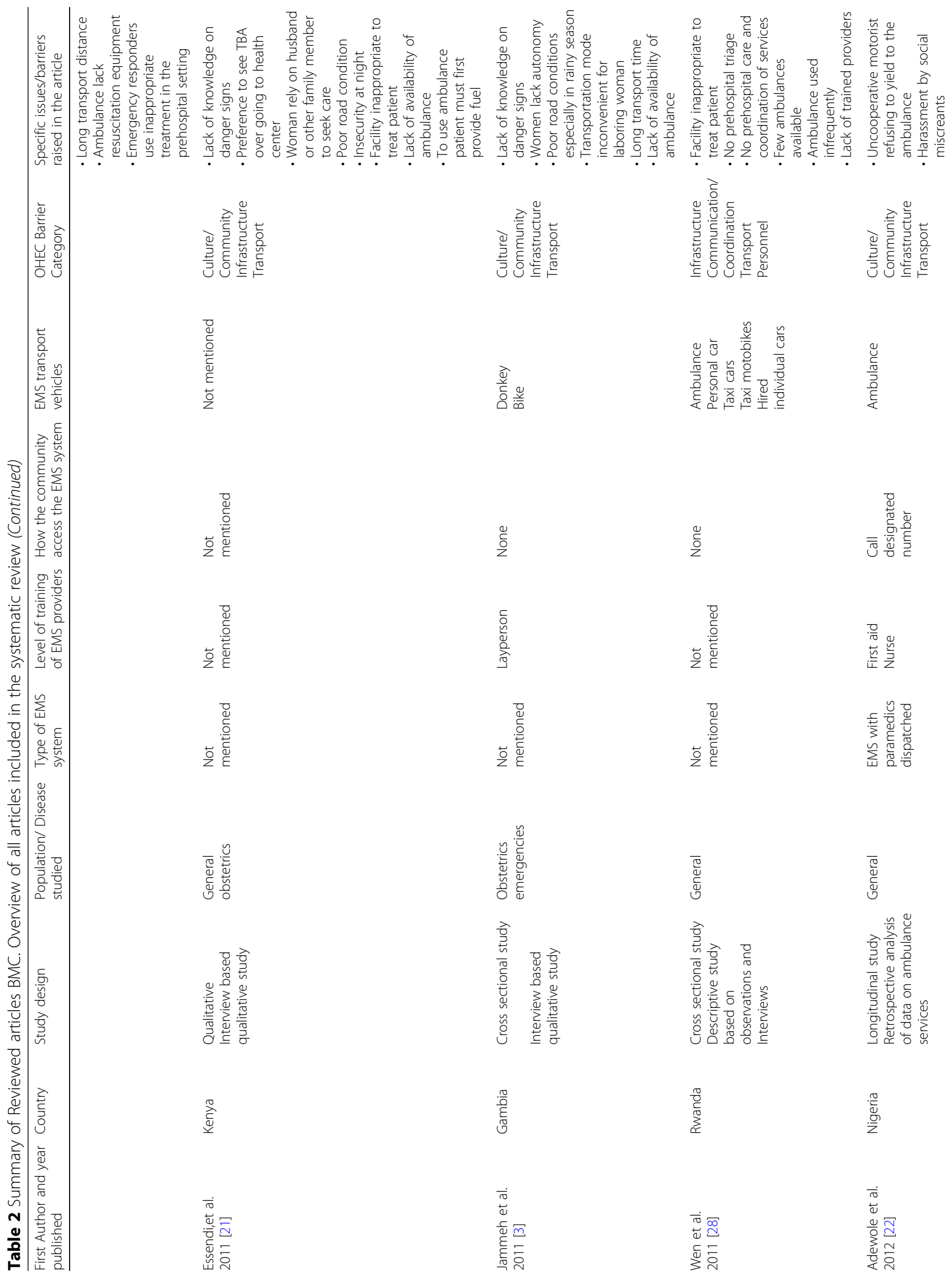




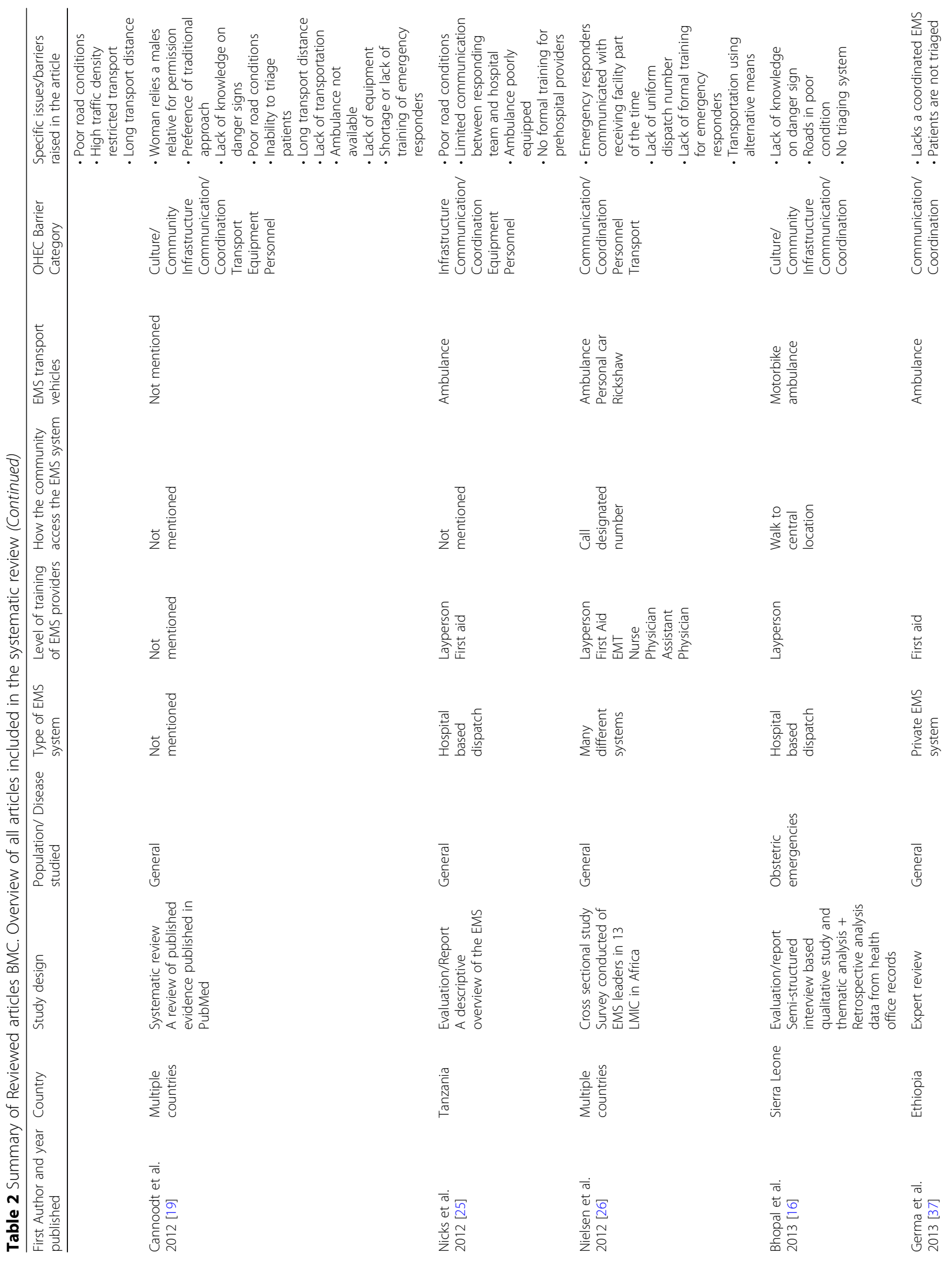




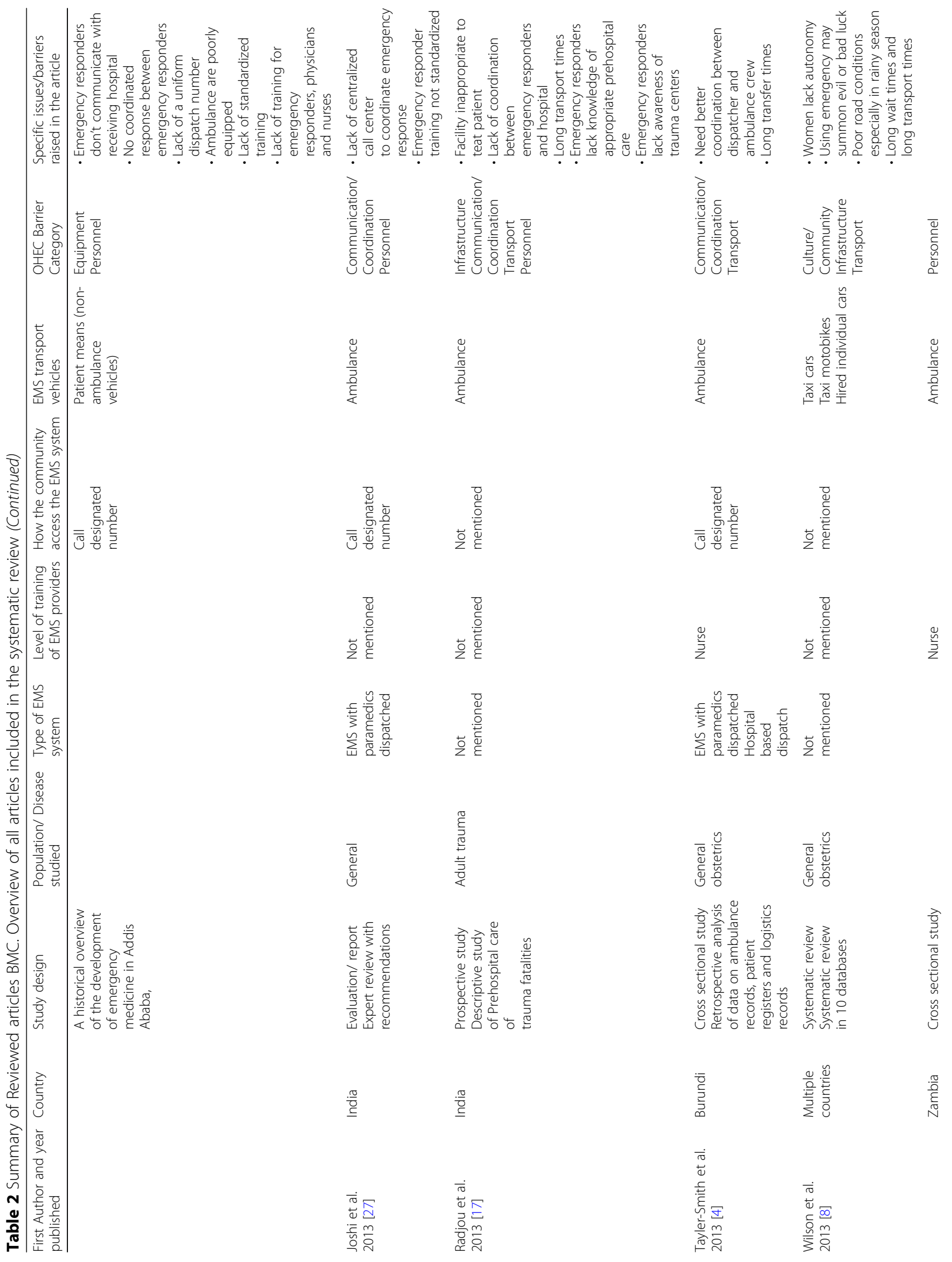




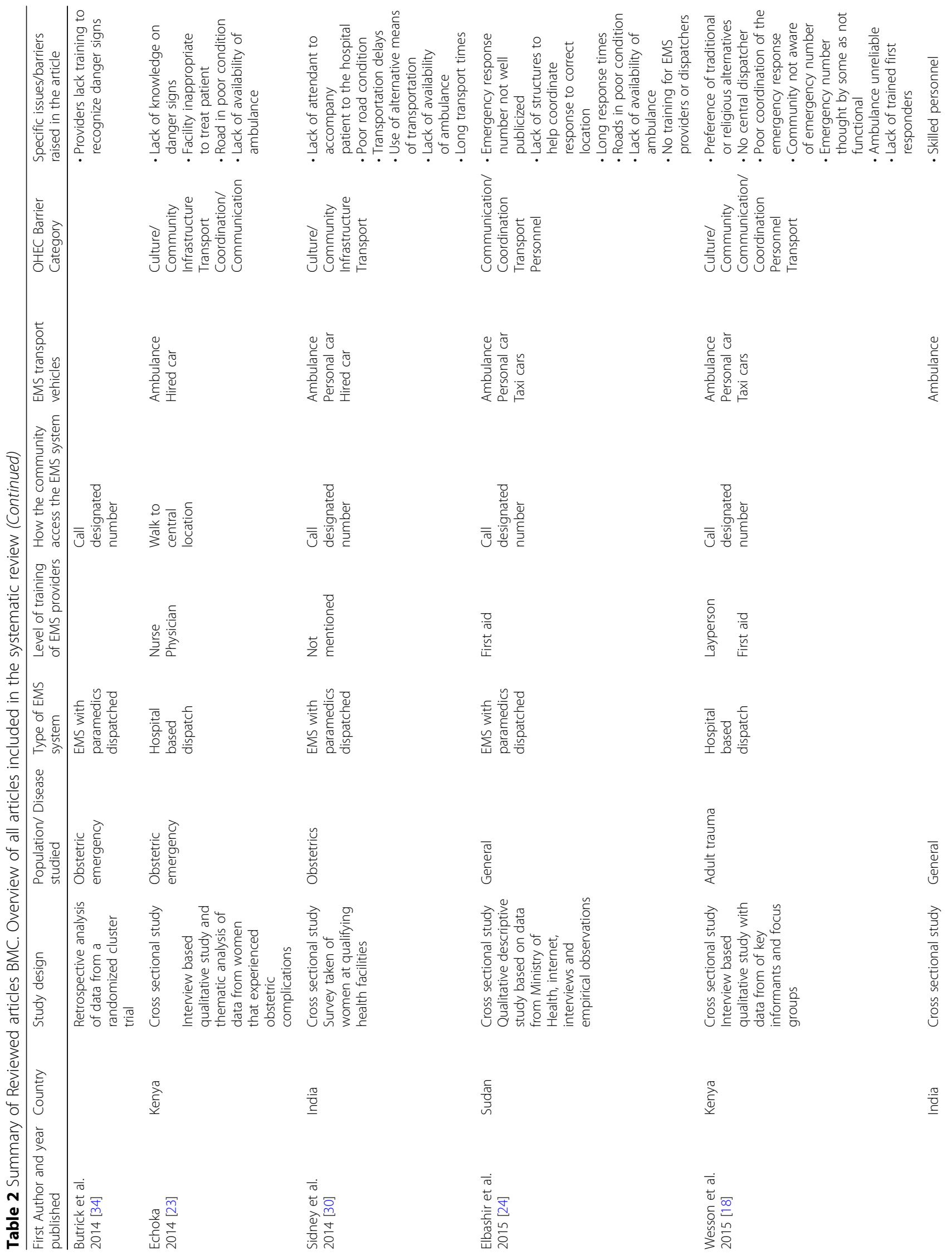




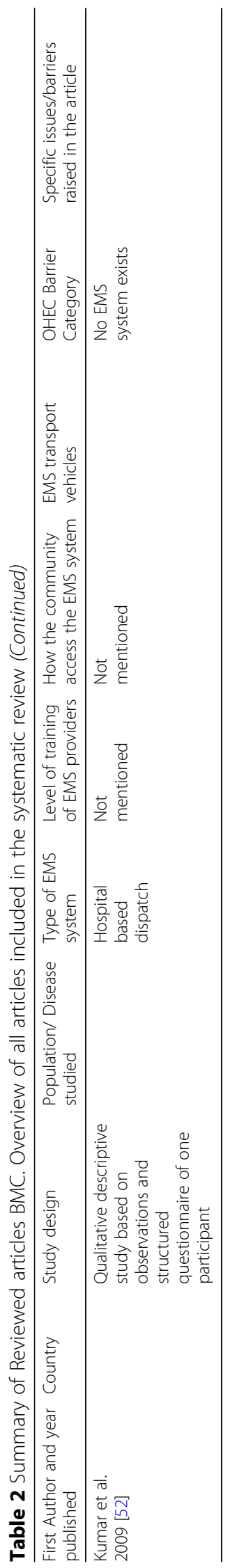




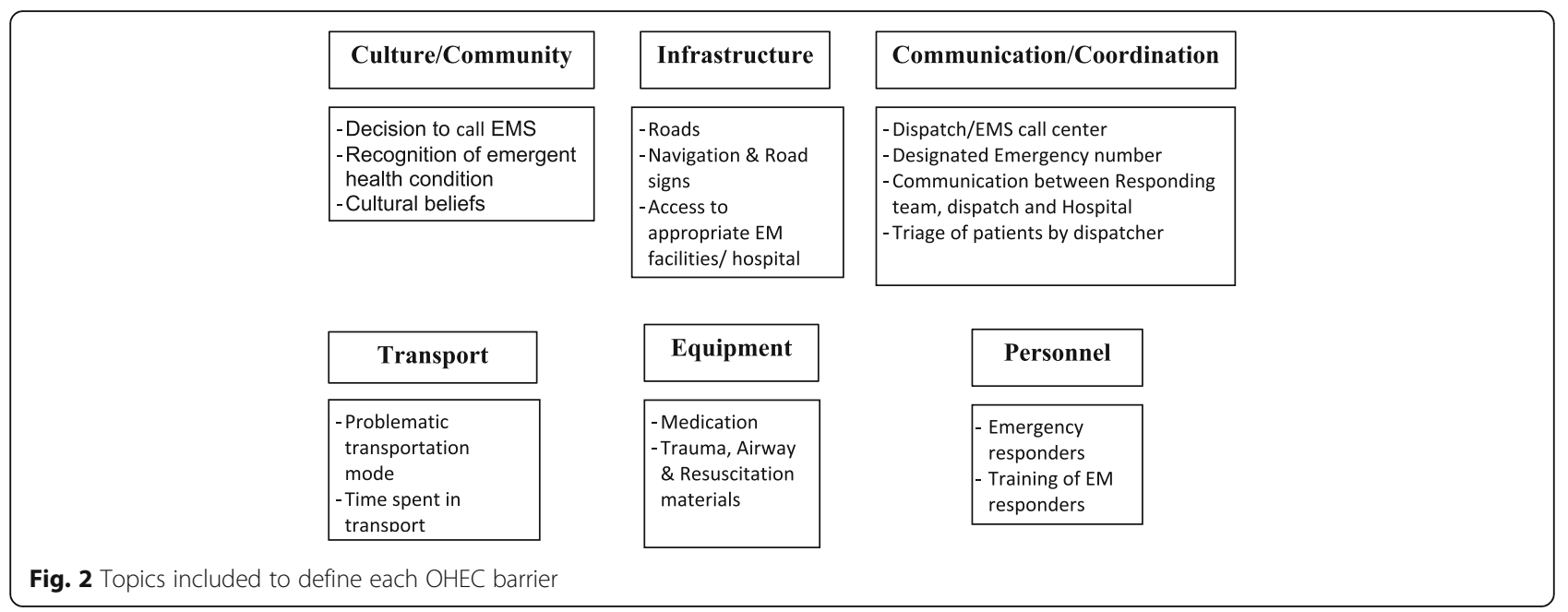

first treated by a facility that did not match the required level of care and $47 \%(8 / 17)$ reported that an emergency medical center was not available. Articles also raised concerns about safety and security preventing patients from seeking care at night [21] or impeding emergency responders' ability to attend to patients [22].

\section{Communication/coordination}

Thirty-nine percent (12/31) described a designated phone number that care seekers could call to activate EMS, and 19\% (6/31) specifically mentioned that care seekers had no means of accessing the EMS system. Two papers $(6 \%)$ noted that patients or their loved ones had to walk to the nearest hospital or clinic to get help. Patients also contacted their local doctors, who didn't immediately refer them to an appropriate treatment

Table 3 Summary of OHEC barrier discussed by the articles in the systematic review

\begin{tabular}{ll}
\hline Barriers Type & Number of articles \\
\hline Culture/community & $39 \%(12 / 31)$ \\
Infrastructure & $55 \%(17 / 31)$ \\
Communication/coordination & $45 \%(14 / 31)$ \\
Transport & $55 \%(17 / 31)$ \\
Equipment & $29 \%(9 / 31)$ \\
Personnel & $61 \%(19 / 31)$ \\
Number of Barriers mentioned & \\
6 barriers & $6 \%(2 / 31)$ \\
5 barriers & $13 \%(4 / 31)$ \\
4 barriers & $32 \%(10 / 31)$ \\
3 barriers & $26 \%(8 / 31)$ \\
2 barriers & $10 \%(3 / 31)$ \\
1 barriers & $3 \%(1 / 31)$ \\
\hline barriers & $13 \%(4 / 31)$ \\
\hline
\end{tabular}

centers $[14,25]$. Other barriers that were noted include: lack of awareness of an emergency number, reliability of the number $[18,24]$, a lack of trust that assistance would show, a lack of public confidence in the EMS [18], and lack of a uniform dispatch number [26]. Additionally, due to unreliable caller information it was difficult to respond to the correct location [24]. Also communication between the ambulance team and the receiving hospital was often limited [17, 25, 26] or uncoordinated [4].

Coordination and management of EMS systems was noted as a barrier in nearly half of articles, 48\%, (15/ $31)$. Ambulances were often not available when requested $[3,23]$ or response efforts were duplicated [27]. A quarter of the articles (8/31), identified dispatch/EMS call center as a deficiency in the current EMS system. Deficiencies include, a complete lack or incomplete coverage by the dispatch center, lack of training for the dispatchers, or delays in relaying information [2, 18]. Triage was also identified as a barrier in $19 \%$ of articles (6/31), often not being performed at all $[16,28]$.

\section{Transport}

Most papers, $71 \%(22 / 31)$, reported ambulances as one of the means of transporting patients. However, the use of alternative means such as hired cars $(5 / 31)$, taxis $(8 / 31)$, motorcycles $(3 / 31)$, bicycles $(2 / 31)$, rickshaws $(1 / 31)$, public transport buses $(5 / 31)$, and animal (donkey, horse or ox) drawn carts (4/31) were also present. Among those articles with ambulances present, it was not guaranteed that it was available to transport patients when needed. Lack of fuel, mechanical failure, challenge moving a woman in labor, need to attend to another patient, and prohibitive costs were among the reasons cited for reduced access to ambulances $[2,3,11,12,14,18,19,21,23$, $29,30]$. An Indian study showed that $35 \%$ of patients 
Table 4 Aggregate characteristics of the articles from the review

\begin{tabular}{ll}
\hline Study Design & \\
\hline Observational & $71 \%(22 / 31)$ \\
Reviews & $13 \%(4 / 31)$ \\
Evaluations/reports & $13 \%(4 / 31)$ \\
Qualitative & $3 \%(1 / 31)$ \\
Geographic Focus & \\
Sub-Saharan Africa & $58 \%(18 / 31)$ \\
India & $16 \%(5 / 31)$ \\
Pakistan & $13 \%(4 / 31)$ \\
$\quad$ More than one country & $13 \%(4 / 31)$ \\
Populations Addressed & \\
Urban & $39 \%(12 / 31)$ \\
Rural & $16 \%(5 / 31)$ \\
Both & $39 \%(12 / 31)$ \\
Disease Focus & \\
Medical emergencies & $45 \%(14 / 31)$ \\
Obstetric care & $39 \%(12 / 31)$ \\
Trauma care & $16 \%(5 / 31)$ \\
Type of OHEC system & \\
Hospital-based & $26 \%(8 / 31)$ \\
Paramedic-based & $3 \%(1 / 31)$ \\
Physician-based & $13 \%(4 / 31)$ \\
No organized OHEC & \\
\hline Topic not addressed & \\
\hline
\end{tabular}

were transported to the hospital via ambulance [15, 31]. Many studies also noted the distance and time it took to get the patient to a hospital being a challenge $[2-4,11,15$, $17,22,24,25,32]$. Time of day also mattered, as transportation at night was not considered safe for fear of being carjacked [18].

\section{Equipment}

Equipment used in a trauma response was identified as a barrier by $36 \%(5 / 14)$ of studies. Hospital ambulances lacked things such as burn kits, spine immobilization boards [29, 32], or the equipment didn't function [2]. Airway, 21\% (3/14), resuscitation equipment, 21\%, (3/14) , and appropriate medications, $29 \%$ (4/14), were also lacking. On some occasions healthcare personnel travelling in the ambulance were asked to carry their own equipment [15].

\section{Personnel}

Lack of formal training was common [2, 24, 26, 29, 32, 33]. More than half of the articles, (18/31), noted that the health system lacked skilled personnel, leaving providers unable to recognize either signs of serious illness [14, 16, 34], the need for a higher level of care [14], or unable to manage acute emergencies $[15,17,35]$. Additionally, some patients were harmed by receipt of inappropriate treatment [15, 17]. In health systems that lacked emergency responders, nonmedical personnel like taxi drivers, police, or good Samaritans with little to no training took over the response duties $[18,29,36]$. In one third, $(10 / 31)$, of articles, OHEC was delivered by laypersons with no training, and 29\% (9/31) noted that it was delivered by a person trained in first-aid. Ten percent (3/31) of articles mentioned specifically trained prehospital providers (EMT) being involved with prehospital care (see Table 5). The health system in our review also lacked standards and regulations to guide OHEC services [24, 27].

\section{Risk of Bias}

Of the studies included, six could be evaluated comprehensively using our risk of bias tool $[4,13,23,28,34$, 36 and six could not be assessed for bias because they were review articles or evaluation/report article $[8,9,19$, $25,27,37]$. The remaining articles were studies where most of the domains from our risk of bias tool were not applicable. Overall, most of the studies had believable results (Fig. 3).

\section{Discussion}

This review highlights a lack of standardization in how OHEC was defined and also in how results pertaining to OHEC were reported. Therefore, we adopted concepts from literature to form a framework that divides OHEC into six categories, in a hope to standardize the topics of discussions as we look to improve OHEC in LIC and LMIC.

\section{Culture/community}

Many factors that influence whether an individual will seek care for an emergent health condition are within the locus of the individual and/or community. The main barriers from our review were: lack of knowledge to recognize danger signs, preferences for traditional approaches, and for many women, lack of authority to make ones' own decisions. Across the literature gender differences in seeking and receiving care are well characterized, particularly in cultures where women have lower levels of literacy and social standing in society than men [38]. While these were the most commonly cited barriers relating to culture and community, they are not necessarily the most important ones. Our understanding of such barriers may be limited by the studies being undertaken by foreign led teams; $56 \%$ of the retrieved articles had a primary author from outside of the study country. 
Table 5 Personnel level of training that are discussed in the articles

\begin{tabular}{ll}
\hline Level of Training & \# Articles that Identify Training Level \\
\hline Not mentioned & $35 \%(12 / 31)$ \\
Layperson & $29 \%(10 / 31)$ \\
First Aid & $29 \%(10 / 31)$ \\
Nurse & $18 \%(6 / 31)$ \\
Other & $15 \%(5 / 31)$ \\
EMT & $12 \%(4 / 31)$ \\
Doctor & $9 \%(3 / 31)$ \\
Physician Assistant & $3 \%(1 / 31)$ \\
\hline
\end{tabular}

Note: Some articles mentioned more than one of the options listed above

Community education has been shown to be successful in addressing community-centered barriers to access [39]. Increased ability to recognize danger signs coupled with awareness and trust of health services can lead to increased utilization of medical services [35] and reductions of delays. Bhopal et al. 2013 used community meetings and film to increase community awareness of new medical services [16]. Increased emphasis on education of women may also help to address gaps in care between men and women. Additionally, working and collaborating with traditional healthcare providers has shown to be beneficial for patients [40]. Moreover, efforts to encourage community members to attend the health facilities for routine and preventative care can also help to familiarize them with the providers and a culture of care delivery [41]. Further research is required, specifically on how community structures can impact service utilization and the barriers that may result.

\section{Infrastructure}

As expected, poor roads that were unreliable - especially during bad weather - played a large role in determining access to care by causing significant delays to patient transport. Further delays also result when patients are taken to facilities that cannot meet their care needs. While many of the barriers in infrastructure noted may be common in rural as well as urban areas, poor roads and scarcity of health facilities are more common in rural areas. However, living in an urban area doesn't guarantee better access to transport or facility infrastructure, as roads are likely more congested [32] and health centers overcrowded $[42,43]$.

It is estimated that mortality increases by $2 \%$ with every $10 \%$ increase in distance traveled to get to a health facility [10]. Thus innovative solutions such a motorcycle transport systems may provide a temporary solution, especially in areas where road quality is not amenable to ambulance transport [13, 16]. Also with navigation affecting transport time [35, 44], innovative solutions that utilize mobile phone and location services may improve time to reaching the patient.

\section{Communication/coordination}

Patients are often very limited in their ability to call for help; 39\% of the health systems described (12/31) had a designated emergency phone number. Out of 178 countries surveyed by the WHO, 56 (31\%) did not have such numbers [45]. Absence of a uniform number adds an extra hurdle when anxiety and worry are high; having a centralized number is a more efficient means of activating the EMS system [46]. Coordination between the different service providers was also noted to be a formidable challenge. A lack of coordination in a health system where resources are scarce can lead to misallocation of resources or in patients not receiving the appropriate level of care. Multifactorial causes are responsible including: lack of coordination between emergency responders and hospitals, lack of training and limited ability to provide care during long transports and scarcity of higher level care centers.

Coordination can be improved by a centralized EMS system with a regional/local coordinating office. This office would be governed by a committee of representatives from health facilities, transportation authorities, local government administration and community representatives [9] to ensure active participation of all stakeholders. Using this as the backbone of the emergency response system, services like a uniform emergency access telephone number and coordination between ambulance services that currently exist can then function more effectively. Better coordination between parties involved in giving OHEC can lead to improved health outcomes and may also lead to less reliance on expensive technology-intensive models like those of high-income countries [35].

\section{Transportation}

Ambulances were the most commonly cited means of transporting patients, but not the most accessible means of transportation. In one study, less than $1 \%$ of the population had access to an ambulance [8]. In highincome countries ambulance transport allows for the emergency responders to quickly get the patient to a treatment center; in LICs and LMICs use of ambulances may not provide the extra benefits of rapid transport, as other motorists are often uncooperative and refuse to give way [22]. However, even when transport is slow, when the ambulance arrives at the hospital the patients are likely attended to more quickly by healthcare providers [34].

In areas with poor road infrastructure, motorbike ambulances have been proposed as better alternatives to vehicles $[8,13,16]$. While motorcycles provide cheaper 


\begin{tabular}{|c|c|c|c|c|c|c|c|c|c|c|c|c|c|}
\hline & 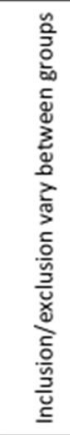 & 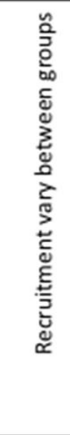 & 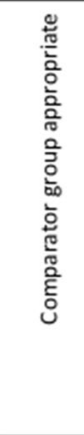 & 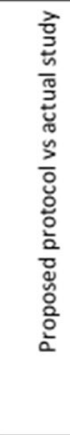 & 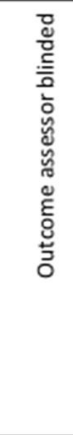 & 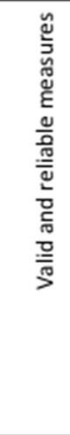 & 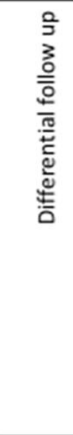 & 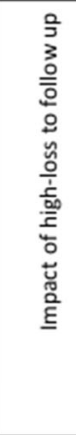 & 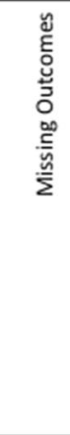 & 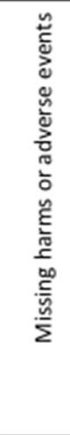 & 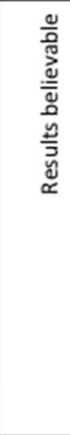 & 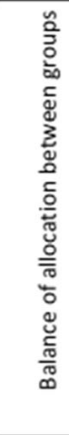 & 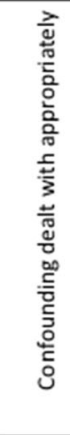 \\
\hline Adewole 2012 & $?$ & $?$ & $?$ & $?$ & $?$ & $?$ & $?$ & $?$ & $\oplus$ & $?$ & $\theta$ & $?$ & $\oplus$ \\
\hline Ali 2006 & $?$ & $?$ & $?$ & $?$ & $?$ & 0 & $?$ & $?$ & $\theta$ & $?$ & $\theta$ & $?$ & 0 \\
\hline Bhopal 2013 & $?$ & $?$ & $?$ & $?$ & $?$ & $\oplus$ & $?$ & $?$ & $\oplus$ & $?$ & $\oplus$ & $?$ & \\
\hline Butrick 2014 & $\theta$ & $\oplus$ & $\theta$ & $\theta$ & $?$ & $\theta$ & $\theta$ & $?$ & $\theta$ & 0 & $\theta$ & $?$ & $\theta$ \\
\hline Cannoodt 2012 & $?$ & $?$ & $?$ & $?$ & $?$ & $\theta$ & $?$ & $?$ & $?$ & $?$ & $?$ & $?$ & $?$ \\
\hline Cham 2005 & $\theta$ & $\theta$ & $?$ & $?$ & $?$ & $?$ & $?$ & $?$ & $?$ & $?$ & $\theta$ & $?$ & 0 \\
\hline Echoka 2014 & $\theta$ & $\oplus$ & $?$ & $\theta$ & 0 & 0 & $\theta$ & $?$ & 0 & 0 & $\theta$ & 0 & 0 \\
\hline Elbashir 2014 & $?$ & $?$ & $?$ & $?$ & $?$ & $?$ & $?$ & $?$ & $\theta$ & $?$ & $\theta$ & $?$ & $\theta$ \\
\hline Essendi 2011 & $?$ & $\theta$ & $?$ & $?$ & $?$ & $\theta$ & $?$ & $?$ & $?$ & $?$ & $\theta$ & $?$ & 0 \\
\hline Germa 2013 & $?$ & $?$ & $?$ & $?$ & $?$ & $?$ & $?$ & $?$ & $?$ & $?$ & $?$ & $?$ & $?$ \\
\hline Hofman 2008 & 0 & 0 & 0 & $\theta$ & 0 & $\oplus$ & $\theta$ & $?$ & 0 & 0 & $\theta$ & $?$ & 0 \\
\hline Jammeh 2011 & $?$ & $?$ & $?$ & $?$ & $?$ & $?$ & $?$ & $?$ & $\theta$ & $?$ & $\theta$ & $?$ & $\theta$ \\
\hline Jayaraman 2009 & $?$ & $?$ & $?$ & $?$ & $?$ & $\oplus$ & $?$ & $?$ & $\theta$ & $?$ & $\theta$ & $?$ & $\theta$ \\
\hline Joshi 2013 & $?$ & $?$ & $?$ & $?$ & $?$ & 0 & $?$ & $?$ & $?$ & $?$ & $\theta$ & $?$ & 0 \\
\hline Khan 2010 & $?$ & $?$ & $?$ & $?$ & $?$ & $\oplus$ & $?$ & $?$ & $\oplus$ & $?$ & $\theta$ & $?$ & 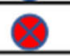 \\
\hline Kobusingye 2005 & $?$ & $?$ & $?$ & $?$ & $?$ & $?$ & $?$ & $?$ & $?$ & $?$ & $?$ & $?$ & $?$ \\
\hline Kumar 2009 & $?$ & $?$ & $?$ & $?$ & $?$ & $\oplus$ & $?$ & $?$ & $\oplus$ & $?$ & $\theta$ & $\theta$ & $\oplus$ \\
\hline Kuwuwa 2007 & $?$ & $?$ & $\theta$ & $?$ & $?$ & $?$ & $?$ & $?$ & $\oplus$ & $?$ & $\theta$ & $?$ & $\oplus$ \\
\hline Macharia 2009 & $\theta$ & $\oplus$ & $?$ & $\theta$ & $?$ & $\oplus$ & $\theta$ & $?$ & $\oplus$ & $?$ & $\theta$ & $?$ & 0 \\
\hline Mahmood 2010 & $?$ & $?$ & $?$ & $?$ & $?$ & $\oplus$ & $\theta$ & $?$ & $?$ & $?$ & $\theta$ & $?$ & 0 \\
\hline Nicks 2012 & $?$ & $?$ & $?$ & $?$ & $?$ & $?$ & $?$ & $?$ & $\theta$ & $?$ & $\theta$ & $?$ & $\theta$ \\
\hline Nielsen 2012 & 0 & 0 & $?$ & $?$ & $?$ & $\theta$ & $?$ & $?$ & $?$ & $?$ & $\theta$ & 0 & $\theta$ \\
\hline Radjou 2013 & $?$ & $?$ & $?$ & $?$ & $?$ & $?$ & $?$ & $?$ & $\theta$ & $?$ & 8 & $?$ & $\theta$ \\
\hline Roy 2010 & $?$ & $?$ & $?$ & $?$ & $?$ & $?$ & $?$ & $?$ & $\oplus$ & $?$ & $\theta$ & $?$ & $\oplus$ \\
\hline Siddiqui 2008 & $?$ & $?$ & $?$ & $?$ & $?$ & $\oplus$ & $?$ & $?$ & $?$ & $?$ & $\theta$ & $?$ & 0 \\
\hline Sidney 2014 & $\theta$ & $\oplus$ & $?$ & $\theta$ & $?$ & $\theta$ & $?$ & $?$ & $?$ & $?$ & $\theta$ & $\theta$ & $\oplus$ \\
\hline Tayler-Smith 2013 & $\oplus$ & $\oplus$ & $\theta$ & $?$ & 0 & 0 & $\theta$ & 0 & 0 & O & $\oplus$ & 0 & $\oplus$ \\
\hline Thompson 2005 & $?$ & $?$ & $?$ & $?$ & $?$ & 0 & $?$ & $?$ & $\theta$ & $?$ & $\theta$ & $?$ & 0 \\
\hline Wen 2011 & $\theta$ & $\theta$ & $?$ & $\theta$ & $?$ & $\theta$ & $\theta$ & $?$ & $\theta$ & $?$ & $\theta$ & $?$ & $?$ \\
\hline Wesson 2015 & $?$ & $?$ & $?$ & $?$ & $?$ & $?$ & $?$ & $?$ & $\theta$ & $?$ & $\theta$ & $?$ & $\theta$ \\
\hline Wilson 2013 & $?$ & $?$ & $?$ & $?$ & $?$ & $?$ & $?$ & $?$ & $\oplus$ & $?$ & $\oplus$ & $?$ & $\oplus$ \\
\hline
\end{tabular}

Fig. 3 Risk of bias summary. Legend: $\bigoplus$ Study met criteria.

Study didn't meet criteria.? Not applicable

alternatives for the rapid transportation of patients, they lack in availability of equipment to stabilize and resuscitate patients during transport. Given that motorcycles are significantly cheaper and ubiquitous in many LIC and LMIC, it may be feasible to increase the number of dispatch sites and to supplement current transport with motorcycle ambulances for low acuity patients [13, 18]. Another strategy is to create partnerships between 
health centers and local transport owners so they too can participate in transporting patients $[3,11]$.

\section{Equipment}

Lack of equipment prevents emergency responders from giving appropriate care to patients; a Mexican study noted that many patients found in respiratory distress did not get appropriate care due to a lack of equipment [47]. This is in sharp contrast, to the well-equipped ambulances seen in most high-income countries [48]. Furthermore, in LICs and LMICs the personnel knowledge and skill level may not match the equipment available [9].

A list of essential prehospital equipment should be developed; personnel responsible for prehospital care should be trained in the proper utilization of that equipment [49]. A sustainable method of acquiring and replenishing the equipment should be established.

\section{Personnel}

Overwhelmingly, a lack of training for the prehospital providers was identified as a barrier. Few health systems had standards and regulations to guide OHEC services. In settings where emergency responders did not exist, untrained bystanders attempt to give care and even transport patients to health centers. In high-income settings where the health system is more formally organized, bystanders primarily serve the role of activating EMS.

The primary focus of training should be on basic livesaving skills [9]. Training can also help to reduce the risk of secondary injuries sustained during the prehospital period [18]. Additionally, training of essential personnel like taxi drivers and the police in basic life support is recommended [29]. Lastly, laypeople trained in basic first-aid skills have been shown to help improve patient outcomes in several settings $[50,51]$.

\section{Finances}

As has been illustrated by previous studies, a lack of financial resources is commonly cited as a significant barrier to OHEC development, including both individual financial barriers and system funding. Our review did not go in depth on these barriers. However, a recent systematic review identified six barriers and proposed a cost sharing model as a way to help bridge the gap in OHEC [19] even though the model may put financial pressures on individuals, sharing the cost between the whole community lessens the burden while giving access to important OHEC to all $[8,16]$. All other barriers presented in this review must be seen in the context of underpinning financial challenges.

\section{Limitations}

This review is restricted to English peer-reviewed literature and thus sustentative descriptions of OHEC in the grey literature or in policy statements may be overlooked. Additionally, our search did not yield studies that featured OHEC in Latin America, South America, Western Europe or Southeast Asia. This exclusion limits the generalizability of the results. Additionally, while most articles had results that were believable, it was difficult to assess bias given the heterogeneity of the studies.

\section{Conclusion}

Policy makers and researchers seeking to improve OHEC care should focus on improving access, transport, and the availability of trained providers. It is important for leaders in OHEC development to perform feasibility analyses to systematically review deficits in the current OHEC system and the potential solutions to address them. Financial barriers undoubtedly impact resource availability and future efforts to develop tools that can prioritize the components of OHEC most impacting a local community's ability to deliver OHEC will be central to these efforts.

\section{Appendix 1 \\ Search Strategy \\ Pubmed Search}

("barrier"[tw] OR "barriers"[tw] OR "delay"[tw] OR "delays"[tw] OR "lack of funding"[tw] OR "lack of communication"[tw] OR "poor roads"[tw] OR "public awareness"[tw] OR "improved access"[tw] OR "insufficient health care"[tw] OR "insufficient healthcare"[tw] OR "lack of equipment"[tw] OR "lack of supplies"[tw] OR "infrastructure"[tw] OR "inadequate funding"[tw] OR "inadequate communication" $[\mathrm{tw}]$ OR "inadequate roads"[tw] OR "inadequate access"[tw] OR "inadequate health care" $[\mathrm{tw}]$ OR "inadequate healthcare"[tw] OR "inadequate equipment"[tw] OR "inadequate supplies"[tw] OR "lack of training"[tw] OR "inadequate training"[tw] OR "local support"[tw] OR "health care access"[tw] OR "healthcare access"[tw] OR "lack of resources"[tw] OR "inadequate resources"[tw]) AND

("Emergency Medical Services"[Mh:noexp] OR "emergency medical services" OR "emergency health service" OR "EMS" OR "emergency medical service" OR "Advanced Trauma Life Support Care"[Mesh] OR "advanced trauma life support" OR "Emergency Medical Service Communication Systems"[Mesh] OR "emergency medical service communication systems" OR "Transportation of Patients"[Mesh] OR "transportation of patients" OR "patient transportation" OR "patient transport" OR "Ambulances"[Mesh] OR "ambulances" OR "ambulance" OR "emergency mobile unit" Or "emergency mobile units" OR "Air Ambulances"[Mesh] OR "air ambulances" OR "air medical transport" OR "emergency helicopter" OR "emergency helicopter" OR "life flight" OR 
"Triage"[Mesh] OR "triage" OR "prehospital" OR "prehospital" OR "EMT" OR "emergency medical technician" OR "emergency medical technicians" OR "paramedic" OR "paramedics" OR "dispatcher" OR "dispatch") AND

\section{Combine: (\#1 OR \#2 OR \#3 OR \#4 OR \#5 OR \#6 OR \#7)}

\#1 Search "developing country"[tiab] OR "developing countries"[tiab] OR "developing nation"[tiab] OR "developing nations"[tiab] OR "developing population"[tiab] OR "developing populations"[tiab] OR "developing world"[tiab] OR "less developed country"[tiab] OR "less developed countries"[tiab] OR "less developed nation"[tiab] OR "less developed nations"[tiab] OR "less developed population"[tiab] OR "less developed populations"[tiab] OR "less developed world"[tiab] OR "lesser developed country"[tiab] OR "lesser developed countries"[tiab] OR "lesser developed nation"[tiab] OR "lesser developed nations"[tiab] OR "lesser developed population"[tiab] OR "lesser developed populations"[tiab] OR "lesser developed world"[tiab] OR "under developed country"[tiab] OR "under developed countries"[tiab] OR "under developed nation"[tiab] OR "under developed nations"[tiab] OR "under developed population"[tiab] OR "under developed populations"[tiab] OR "under developed world"[tiab] OR "underdeveloped country"[tiab] OR "underdeveloped countries"[tiab] OR "underdeveloped nation"[tiab] OR "underdeveloped nations"[tiab] OR "underdeveloped population"[tiab] OR "underdeveloped populations"[tiab] OR "underdeveloped world"[tiab] OR "middle income country"[tiab] OR "middle income countries"[tiab] OR "middle income nation"[tiab] OR "middle income nations"[tiab] OR "middle income population"[tiab] OR "middle income populations"[tiab] OR "low income country"[tiab] OR "low income countries"[tiab] OR "low income nation"[tiab] OR "low income nations"[tiab] OR "low income population"[tiab] OR "low income populations"[tiab] OR "lower income country"[tiab] OR "lower income countries"[tiab] OR "lower income nation"[tiab] OR "lower income nation$\mathrm{s}$ "[tiab] OR "lower income population"[tiab] OR "lower income populations"[tiab] OR "underserved country"[tiab] OR "underserved countries"[tiab] OR "underserved nation"[tiab] OR "underserved nations"[tiab] OR "underserved population"[tiab] OR "underserved population$\mathrm{s}$ "[tiab] OR "underserved world"[tiab] OR "under served country"[tiab] OR "under served countries"[tiab] OR "under served nation"[tiab] OR "under served nation$s$ "[tiab] OR "under served population"[tiab] OR "under served populations"[tiab] OR "under served world"[tiab] OR "deprived country"[tiab] OR "deprived countries"[tiab] OR "deprived nation"[tiab] OR "deprived nation$s "[$ tiab] OR "deprived population"[tiab] OR "deprived populations"[tiab] OR "deprived world"[tiab]
\#2 Search "poor country"[tiab] OR "poor countries"[tiab] OR "poor nation"[tiab] OR "poor nations"[tiab] OR "poor population"[tiab] OR "poor populations"[tiab] OR "poor world"[tiab] OR "poorer country"[tiab] OR "poorer countries"[tiab] OR "poorer nation"[tiab] OR "poorer nations"[tiab] OR "poorer population"[tiab] OR "poorer populations"[tiab] OR "poorer world"[tiab] OR "developing economy"[tiab] OR "developing economies"[tiab] OR "less developed economy"[tiab] OR "less developed economies"[tiab] OR "lesser developed economy"[tiab] OR "lesser developed economies"[tiab] OR "under developed economy"[tiab] OR "under developed economies"[tiab] OR "underdeveloped economy"[tiab] OR "underdeveloped economies"[tiab] OR "middle income economy"[tiab] OR "middle income economies"[tiab] OR "low income economy"[tiab] OR "low income economies"[tiab] OR "lower income economy"[tiab] OR "lower income economies"[tiab] OR "low gdp"[tiab] OR "low gnp"[tiab] OR "low gross domestic"[tiab] OR "low gross national"[tiab] OR "lower gdp"[tiab] OR "lower gnp"[tiab] OR "lower gross domestic"[tiab] OR "lower gross national"[tiab] OR lmic[tiab] OR lmics[tiab] OR "third world"[tiab] OR "lami country"[tiab] OR "lami countries"[tiab] OR "transitional country"[tiab] OR "transitional countries"[tiab]

\#3 Search Africa[tiab] OR Asia[tiab] OR Caribbean[tiab] OR West Indies[tiab] OR South America[tiab] OR Latin America[tiab] OR Central America[tiab] OR "Atlantic Islands"[tiab] OR "Commonwealth of Independent States"[tiab] OR "Pacific Islands"[tiab] OR "Indian Ocean Islands"[tiab] OR "Eastern Europe"[tiab] OR Afghanis$\tan [$ tiab] OR Albania[tiab] OR Algeria[tiab] OR Angola[tiab] OR Antigua[tiab] OR Barbuda[tiab] OR Argentina[tiab] OR Armenia[tiab] OR Armenian[tiab] OR Aruba[tiab] OR Azerbaijan[tiab] OR Bahrain[tiab] OR Bangladesh[tiab] OR Barbados[tiab] OR Benin[tiab] OR Byelarus[tiab] OR Byelorussian[tiab] OR Belarus[tiab] OR Belorussian[tiab] OR Belorussia[tiab] OR Belize[tiab] OR Bhutan[tiab] OR Bolivia[tiab] OR Bosnia[tiab] OR Herzegovina[tiab] OR Hercegovina[tiab] OR Botswana[tiab] OR Brasil[tiab] OR Brazil[tiab] OR Bulgaria[tiab] OR Burkina Faso[tiab] OR Burkina Fasso[tiab] OR Upper Volta[tiab] OR Burundi[tiab] OR Urundi[tiab] OR Cambodia[tiab] OR Khmer Republic[tiab] OR Kampuchea[tiab] OR Cameroon[tiab] OR Cameroons[tiab] OR Cameron[tiab] OR Camerons[tiab] OR Cape Verde[tiab] OR Central African Republic[tiab] OR Chad[tiab] OR Chile[tiab] OR China[tiab] OR Colombia[tiab] OR Comoros[tiab] OR Comoro Islands[tiab] OR Comores[tiab] OR Mayotte[tiab] OR Congo[tiab] OR Zaire[tiab] OR Costa Rica[tiab] OR Cote d'Ivoire[tiab] OR Ivory Coast[tiab] OR Croatia[tiab] OR Cuba[tiab] OR Cyprus[tiab] OR Czechoslovakia[tiab] OR Czech Republic[tiab] OR Slovakia[tiab] OR Slovak 
Republic[tiab] OR Djibouti[tiab] OR French Somaliland[tiab] OR Dominica[tiab] OR Dominican Republic[tiab] OR East Timor[tiab] OR East Timur[tiab] OR Timor Leste[tiab] OR Ecuador[tiab] OR Egypt[tiab] OR United Arab Republic[tiab] OR El Salvador[tiab] OR Eritrea[tiab] OR Estonia[tiab] OR Ethiopia[tiab] OR Fiji[tiab] OR Gabon[tiab] OR Gabonese Republic[tiab] OR Gambia[tiab] OR Gaza[tiab] OR Georgia Republic[tiab] OR Georgian Republic[tiab] OR Ghana[tiab] OR Gold Coast[tiab] OR Greece[tiab] OR Grenada[tiab] OR Guatemala[tiab] OR Guinea[tiab] OR Guam[tiab] OR Guiana[tiab] OR Guyana[tiab] OR Haiti[tiab] OR Honduras[tiab] OR Hungary[tiab] OR India[tiab] OR Maldives[tiab] OR Indonesia[tiab] OR Iran[tiab] OR Iraq[tiab] OR Isle of Man[tiab] OR Jamaica[tiab] OR Jordan[tiab] OR Kazakhstan[tiab] OR Kazakh[tiab] OR Kenya[tiab] OR Kiribati[tiab] OR Korea[tiab] OR Kosovo[tiab] OR Kyrgyzstan[tiab] OR Kirghizia[tiab] OR Kyrgyz Republic[tiab] OR Kirghiz[tiab] OR Kirgizstan[tiab] OR "Lao PDR"[tiab] OR Laos[tiab] OR Latvia[tiab] OR Lebanon[tiab] OR Lesotho[tiab] OR Basutoland[tiab] OR Liberia[tiab] OR Libya[tiab] OR Lithuania[tiab]

\#4 Search Macedonia[tiab] OR Madagascar[tiab] OR Malagasy Republic[tiab] OR Malaysia[tiab] OR Malaya[tiab] OR Malay[tiab] OR Sabah[tiab] OR Sarawak[tiab] OR Malawi[tiab] OR Nyasaland[tiab] OR Mali[tiab] OR Malta[tiab] OR Marshall Islands[tiab] OR Mauritania[tiab] OR Mauritius[tiab] OR Agalega Islands[tiab] OR "Melanesia"[tiab] OR Mexico[tiab] OR Micronesia[tiab] OR Middle East[tiab] OR Moldova[tiab] OR Moldovia[tiab] OR Moldovian[tiab] OR Mongolia[tiab] OR Montenegro[tiab] OR Morocco[tiab] OR Ifni[tiab] OR Mozambique[tiab] OR Myanmar[tiab] OR Myanma[tiab] OR Burma[tiab] OR Namibia[tiab] OR Nepal[tiab] OR Netherlands Antilles[tiab] OR New Caledonia[tiab] OR Nicaragua[tiab] OR Niger[tiab] OR Nigeria[tiab] OR Northern Mariana Islands[tiab] OR Oman[tiab] OR Muscat[tiab] OR Pakistan[tiab] OR Palau[tiab] OR Palestine[tiab] OR Panama[tiab] OR Paraguay[tiab] OR Peru[tiab] OR Philippines[tiab] OR Philipines[tiab] OR Phillipines[tiab] OR Phillippines[tiab] OR Poland[tiab] OR Portugal[tiab] OR Puerto Rico[tiab] OR Romania[tiab] OR Rumania[tiab] OR Roumania[tiab] OR Russia[tiab] OR Russian[tiab] OR Rwanda[tiab] OR Ruanda[tiab] OR Saint Kitts[tiab] OR St Kitts[tiab] OR Nevis[tiab] OR Saint Lucia[tiab] OR St Lucia[tiab] OR Saint Vincent[tiab] OR St Vincent[tiab] OR Grenadines[tiab] OR Samoa[tiab] OR Samoan Islands[tiab] OR Navigator Island[tiab] OR Navigator Islands[tiab] OR Sao Tome[tiab] OR Saudi Arabia[tiab] OR Senegal[tiab] OR Serbia[tiab] OR Montenegro[tiab] OR Seychelles[tiab] OR Sierra Leone[tiab] OR Slovenia[tiab] OR Sri Lanka[tiab] OR Ceylon[tiab] OR Solomon Islands[tiab] OR Somalia[tiab] OR Sudan[tiab] OR Suriname[tiab]
OR Surinam[tiab] OR Swaziland[tiab] OR Syria[tiab] OR Syrian[tiab] OR Tajikistan[tiab] OR Tadzhikistan[tiab] OR Tadjikistan[tiab] OR Tadzhik[tiab] OR Tanzania[tiab] OR Thailand[tiab] OR Togo[tiab] OR Togolese Republic[tiab] OR Tonga[tiab] OR Trinidad[tiab] OR Tobago[tiab] OR Tunisia[tiab] OR Turkey[tiab] OR Turkmenistan[tiab] OR Turkmen[tiab] OR Tuvalu[tiab] OR Uganda[tiab] OR Ukraine[tiab] OR Uruguay[tiab] OR USSR[tiab] OR Soviet Union[tiab] OR Union of Soviet Socialist Republics[tiab] OR Uzbekistan[tiab] OR Uzbek OR Vanuatu[tiab] OR New Hebrides[tiab] OR Venezuela[tiab] OR Vietnam[tiab] OR Viet Nam[tiab] OR West Bank[tiab] OR Yemen[tiab] OR Yugoslavia[tiab] OR Zambia[tiab] OR Zimbabwe[tiab] OR Rhodesia[tiab]

\#5 Search Developing Countries[Mesh] OR Africa[Mesh:NoExp] OR Africa, Northern[Mesh:NoExp] OR Africa South of the Sahara[Mesh:NoExp] OR Africa, Central[Mesh:NoExp] OR Africa, Eastern[Mesh:NoExp] OR Africa, Southern[Mesh:NoExp] OR Africa, Western[Mesh:NoExp] OR Asia[Mesh:NoExp] OR Asia, Central[Mesh:NoExp] OR Asia, Southeastern[Mesh: NoExp] OR Asia, Western[Mesh:NoExp] OR Caribbean Region[Mesh:NoExp] OR West Indies[Mesh:NoExp] OR South America[Mesh:NoExp] OR Latin America[Mesh: NoExp] OR Central America[Mesh:NoExp] OR "Atlantic Islands"[Mesh:NoExp] OR "Commonwealth of Independent States"[Mesh:NoExp] OR "Pacific Islands"[Mesh: NoExp] OR "Indian Ocean Islands"[Mesh:NoExp] OR "Europe, Eastern"[Mesh:NoExp]

\#6 Search Afghanistan[Mesh] OR Albania[Mesh] OR Algeria[Mesh] OR American Samoa[Mesh] OR Angola[Mesh] OR "Antigua and Barbuda"[Mesh] OR Argentina[Mesh] OR Armenia[Mesh] OR Azerbaijan[Mesh] OR Bahrain[Mesh] OR "Baltic States"[Mesh] OR Bangladesh[Mesh] OR Barbados[Mesh] OR Benin[Mesh] OR "Republic of Belarus"[Mesh] OR Belize[Mesh] OR Bhutan[Mesh] OR Bolivia[Mesh] OR Bosnia-Herzegovina[Mesh] OR Botswana[Mesh] OR Brazil[Mesh] OR Bulgaria[Mesh] OR Burkina Faso[Mesh] OR Burundi[Mesh] OR Cambodia[Mesh] OR Cameroon[Mesh] OR Cape Verde[Mesh] OR Central African Republic[Mesh] OR Chad[Mesh] OR Chile[Mesh] OR China[Mesh] OR Colombia[Mesh] OR Comoros[Mesh] OR Congo[Mesh] OR Costa Rica[Mesh] OR Cote d'Ivoire[Mesh] OR Croatia[Mesh] OR Cuba[Mesh] OR Cyprus[Mesh] OR Czechoslovakia[Mesh] OR Czech Republic[Mesh] OR Slovakia[Mesh] OR Djibouti[Mesh] OR "Democratic Republic of the Congo"[Mesh] OR "Democratic People's Republic of Korea"[Mesh] OR Dominica[Mesh] OR Dominican Republic[Mesh] OR East Timor[Mesh] OR Ecuador[Mesh] OR Egypt[Mesh] OR El Salvador[Mesh] OR Eritrea[Mesh] OR Estonia[Mesh] OR Ethiopia[Mesh] OR "Equatorial Guinea"[Mesh] OR Fiji[Mesh] OR "French Guiana"[Mesh] OR Gabon[Mesh] OR Gambia[Mesh] OR "Georgia (Republic)"[Mesh] OR 
Ghana[Mesh] OR Greece[Mesh] OR Grenada[Mesh] OR Guatemala[Mesh] OR Guinea[Mesh] OR GuineaBissau[Mesh] OR Guam[Mesh] OR Guyana[Mesh] OR Haiti[Mesh] OR Honduras[Mesh] OR Hungary[Mesh] OR "Independent State of Samoa"[Mesh] OR India[Mesh] OR Indonesia[Mesh] OR Iran[Mesh] OR Iraq[Mesh] OR Jamaica[Mesh] OR Jordan[Mesh] OR Kazakhstan[Mesh] OR Kenya[Mesh] OR Korea[Mesh] OR Kyrgyzstan[Mesh] OR Laos[Mesh] OR Latvia[Mesh] OR Lebanon[Mesh] OR Lesotho[Mesh] OR Liberia[Mesh] OR Libya[Mesh] OR Lithuania[Mesh] OR "Macedonia (Republic)"[Mesh] OR Madagascar[Mesh]

\#7 Search Malawi[Mesh] OR Malaysia[Mesh] OR Mali[Mesh] OR Malta[Mesh] OR Mauritania[Mesh] OR Mauritius[Mesh] OR "Melanesia"[Mesh] OR Mexico[Mesh] OR Micronesia[Mesh] OR Middle East[Mesh: NoExp] OR Moldova[Mesh] OR Mongolia[Mesh] OR Montenegro[Mesh] OR Morocco[Mesh] OR Mozambique[Mesh] OR Myanmar[Mesh] OR Namibia[Mesh] OR Nepal[Mesh] OR Netherlands Antilles[Mesh] OR New Caledonia[Mesh] OR Nicaragua[Mesh] OR Niger[Mesh] OR Nigeria[Mesh] OR Oman[Mesh] OR Pakistan[Mesh] OR Palau[Mesh] OR Panama[Mesh] OR Papua New Guinea[Mesh] OR Paraguay[Mesh] OR Peru[Mesh] OR Philippines[Mesh] OR Poland[Mesh] OR Portugal[Mesh] OR Puerto Rico[Mesh] OR "Republic of Korea"[Mesh] OR Romania[Mesh] OR Russia[Mesh] OR "Russia (Pre1917)"[Mesh] OR Rwanda[Mesh] OR "Saint Kitts and Nevis"[Mesh] OR Saint Lucia[Mesh] OR "Saint Vincent and the Grenadines"[Mesh] OR Samoa[Mesh] OR Saudi Arabia[Mesh] OR Senegal[Mesh] OR Serbia[Mesh] OR Montenegro[Mesh] OR Seychelles[Mesh] OR Sierra Leone[Mesh] OR Slovenia[Mesh] OR Sri Lanka[Mesh] OR Somalia[Mesh] OR South Africa[Mesh] OR Sudan[Mesh] OR Suriname[Mesh] OR Swaziland[Mesh] OR Syria[Mesh] OR Tajikistan[Mesh] OR Tanzania[Mesh] OR Thailand[Mesh] OR Togo[Mesh] OR Tonga[Mesh] OR "Trinidad and Tobago"[Mesh] OR Tunisia[Mesh] OR Turkey[Mesh] OR Turkmenistan[Mesh] OR Ugan$\mathrm{da}$ [Mesh] OR Ukraine[Mesh] OR Uruguay[Mesh] OR USSR[Mesh] OR Uzbekistan[Mesh] OR Vanuatu[Mesh] OR Venezuela[Mesh] OR Vietnam[Mesh] OR Yemen[Mesh] OR Yugoslavia[Mesh] OR Zambia[Mesh] OR Zimbabwe[Mesh]

\footnotetext{
Abbreviations

AFEM: African Federation for Emergency Medicine; EMS: Emergency medical services; FRC: First responder care; LIC: Low-income countries; LMIC: Lowmiddle income countries; OHEC: Out of hospital emergency care; PHC: Prehospital care; WHO: World Health Organization
}

\section{Acknowledgements}

This work wouldn't have been completed without the help of Katie Lobner who was pivotal in helping us to design the search strategy for our work.
Availability of data and materials

All data generated or analyzed during this study are included in this published article and its supplementary information files (see Appendix 1: Search Strategy).

\section{Authors' contributions}

The following authors contributed in the study design, data collection, data analysis, data interpretation and writing of the manuscript: $\mathrm{AGK}$ and $\mathrm{BH}$. The following authors contributed in the study design, data collection and writing of the manuscript: PH, SSR and TA. The following authors contributed in the writing of the manuscript: LW, and JR. The following author contributed in the study design, and data collection: AJ. All authors read and approved the final manuscript.

Ethics approval and consent to participate

Not applicable.

\section{Competing interests}

The authors declare that they have no competing interests.

\section{Publisher's Note}

Springer Nature remains neutral with regard to jurisdictional claims in published maps and institutional affiliations.

\section{Author details}

${ }^{1}$ Johns Hopkins School of Medicine, Baltimore, MD, USA. ${ }^{2}$ Division of Emergency Medicine, University of Cape Town, Cape Town, South Africa. ${ }^{3}$ Department of Emergency Medicine, Johns Hopkins Hospital, Baltimore, MD, USA.

Received: 31 August 2016 Accepted: 3 April 2018

Published online: 19 April 2018

\section{References}

1. Kobusingye OC, Hyder AA, Bishai D, Joshipura M, Hicks ER, Mock C: Disease control priorities in developing countries. In Medical services. In: Jamison DT, Breman JG, Measham AR, et al, editors. 2nd edition. Edited by Anonymous Washington (DC): World Bank; 2006:Chapter 68.

2. Thomson N. Emergency medical services in Zimbabwe. Resuscitation. 2005; 65:15-9.

3. Jammeh A, Sundby J, Vangen S. Barriers to emergency obstetric care services in perinatal deaths in rural Gambia: a qualitative in-depth interview study. ISRN Obstet Gynecol. 2011;2011:981096.

4. Tayler-Smith K, Zachariah R, Manzi M, Van den Boogard W, Nyandwi G, Reid T, De Plecker E, Lambert V, Nicolai M, Goetghebuer S, Christiaens B, Ndelema B, Kabangu A, Manirampa J, Harries AD. An ambulance referral network improves access to emergency obstetric and neonatal care in a district of rural Burundi with high maternal mortality. Tropical Med Int Health. 2013;18:993-1001.

5. Calvello E, Reynolds T, Hirshon JM, Buckle C, Moresky R, O'Neill J, Wallis LA. Emergency care in sub-Saharan Africa: results of a consensus conference. Afr J Emerg Med. 2013;3(1):42-8.

6. Mock CN, Jurkovich GJ, Arreola-Risa C, Maier RV. Trauma mortality patterns in three nations at different economic levels: implications for global trauma system development. J Trauma Acute Care Surg. 1998;44(5):804-14.

7. Sasser S, Varghese M, Kellermann A, Lormand J. Prehospital trauma care systems. In: Anonymous, editor. Prehospital trauma care systems. Geneva: World Health Organization; 2005.

8. Wilson A, Hillman S, Rosato M, Skelton J, Costello A, Hussein J, MacArthur C, Coomarasamy A. A systematic review and thematic synthesis of qualitative studies on maternal emergency transport in low-and middle-income countries. Int J Gynecol Obstet. 2013;122(3):192-201.

9. Kobusingye OC, Hyder AA, Bishai D, Hicks ER, Mock C, Joshipura M. Emergency medical systems in low- and middle-income countries: recommendations for action. Bull World Health Organ. 2005;83:626-31.

10. Forster $\mathrm{G}$, Simfukwe $\mathrm{V}$, Barber $\mathrm{C}$. Use of intermediate modes of transport for patient transport: a literature review contrasted with the findings of the Transaid bicycle ambulance project in eastern Zambia. Transaid-Transp Life. 2009;137. https://www.jiscmail.ac.uk/cgi-bin/webadmin?A3=ind0906\&L= RURAL-TRANSPORT-DEVELOPMENT\&E=base64\&P=3369\&B=-part-DolGmxs4R-3RQSqa1apP2mCW-sX7KBgpHbYW2CrqGm-F\&T=APPLICATION\%2FPDF; $\% 20$ name $=\% 22$ Transaid\%20Bicycle\%20Ambulance\%20Paper\%20May \%202009.pdf\%22\&N=Transaid\%20Bicycle\%20Ambulance\%20Paper\%20May \%202009.pdf. 
11. Cham M, Sundby J, Vangen S. Maternal mortality in the rural Gambia, a qualitative study on access to emergency obstetric care. Reprod Health. 2005;2:3.

12. Kawuwa MB, Mairiga AG, Usman HA. Community perspective of maternal mortality: experience from Konduga local government area, Borno state, Nigeria. Ann Afr Med. 2007;6:109-14.

13. Hofman JJ, Dzimadzi C, Lungu K, Ratsma EY, Hussein J. Motorcycle ambulances for referral of obstetric emergencies in rural Malawi: do they reduce delay and what do they cost? Int J Gynaecol Obstet. 2008;102:191-7.

14. Siddiqui M, Siddiqui SR, Zafar A, Khan FS. Factors delaying hospital arrival of patients with acute strokel. J Pak Med Assoc. 2008;58:178-82

15. Roy N, Murlidhar V, Chowdhury R, Patil SB, Supe PA, Vaishnav PD, Vatkar A. Where there are no emergency medical services-prehospital care for the injured in Mumbai, India. Prehospital Disaster Med. 2010;25:145-51.

16. Bhopal SS, Halpin SJ, Gerein N. Emergency obstetric referral in rural Sierra Leone: what can motorbike ambulances contribute? A mixed-methods study. Matern Child Health J. 2013;17:1038-43.

17. Radjou AN, Mahajan P, Baliga DK. Where do I go? A trauma victim's plea in an informal trauma system. J Emerg Trauma Shock. 2013;6:164-70.

18. Wesson HK, Stevens KA, Bachani AM, Mogere S, Akungah D, Nyamari J, Masasabi Wekesa J, Hyder AA. Trauma Systems in Kenya: a qualitative analysis at the district level. Qual Health Res. 2015;

19. Cannoodt L, Mock C, Bucagu M. Identifying barriers to emergency care services. Int J Health Plann Manag. 2012;27:e104-20.

20. Viswanathan M, Berkman ND, Dryden DM, HartlingL. Assessing Risk of Bias and Confounding in Observational Studies of Interventions or Exposures: Further Development of the RTI Item Bank [Internet]. Rockville (MD): Agency for Healthcare Research and Quality (US); 2013. Available from: https://www. ncbi.nlm.nih.gov/books/NBK154461/.

21. Essendi $H$, Mills $S$, Fotso JC. Barriers to formal emergency obstetric care. services' utilization. J Urban Health. 2011;88(Suppl 2):S356-69.

22. Adewole OA, Fadeyibi IO, Kayode MO, Giwa SO, Shoga MO, Adejumo AO, Ademiluyi SA. Ambulance services of Lagos state, Nigeria: a six-year (20012006) audit. West Afr J Med. 2012;31:3-7.

23. Echoka E. Barriers to emergency obstetric care services: accounts of survivors of life threatening obstetric complications in Malindi District, Kenya. Pan Afr Med J. 2014:17:4.

24. Elbashir K, Gore RJ, Abuaaraki T, Roblin P, Botha M, Yousif M, Ostrovskiys G, Bloem C, James SA. Prehospital emergency care and injury prevention in Sudan. Afr J Emerg Med. 2014;4:170-3.

25. Nicks BA, Sawe HR, Juma AM, Reynolds TA. The state of emergency medicine in the United Republic of Tanzania. Afr J Emerg Med. 2012;2:97-102.

26. Nielsen K, Mock C, Joshipura M, Rubiano AM, Zakariah A, Rivara F. Assessment of the status of prehospital care in 13 low- and middle-income countries. Prehosp Emerg Care. 2012;16:381-9.

27. Joshi A, Rajhans P, Galwankar S, Arquilla B, Swaroop M, Stawicki S, Das B, Aggarwal P, Bhoi S, Kalra OP. Academic College of Emergency Experts in India's INDO-US joint working group (JWG) white paper on the integrated emergency communication response Service in India: much more than just a number! J Emerg Trauma Shock. 2013;6:216-23.

28. Wen LS, Char D. Existing infrastructure for the delivery of emergency care in postconflict Rwanda: an initial descriptive study. Acad Emerg Med. 2011;18:S243.

29. Jayaraman S, Mabweijano JR, Lipnick MS, Caldwell N, Miyamoto J, Wangoda R, Mijumbi C, Hsia R, Dicker R, Ozgediz D. Current patterns of prehospital trauma care in Kampala, Uganda and the feasibility of a lay-first-responder training program. World J Surg. 2009;33:2512-21.

30. Sidney K, Ryan K, Diwan V, De Costa A. Utilization of a state run public private emergency transportation service exclusively for childbirth: the Janani (maternal) express program in Madhya Pradesh, India. PLoS One. 2014;9:e96287.

31. Forjouh S, Mock C, Freidman D, Quansah R. Transport of the injured to hospitals in Ghana: the need to strengthen the practice of trauma care. Prehospital Immediate Care. 1999;3(1):66-70.

32. Mahmood KT, Amin F, Ayub H, Yaqoob M, Zaka M. Management of the patient from the site of accident to the hospital/ pre-hospital care. J Pharm Sci Res. 2010;2:804-8.

33. Khan A, Zafar H, Naeem SN, Raza SA. Transfer delay and in-hospital mortality of trauma patients in Pakistan. Int J Surg. 2010;8:155-8.

34. Butrick E, Penn A, Itakura K, Mkumba G, Winter K, Amafumba R, Miller S. Access to transport for women with hypovolemic shock differs according to weeks of pregnancy. Int J Gynecol Obstet. 2014;127:171-4.

35. Ali M, Miyoshi C, Ushijima H. Emergency medical services in Islamabad, Pakistan: a public-private partnership. Public Health. 2006;120:50-7.
36. Macharia WM, Njeru EK, Muli-Musiime F, Nantulya V. Severe road traffic injuries in Kenya, quality of care and access. Afr Health Sci. 2009:9:118-24.

37. Germa F, Bayleyegn T, Kebede T, Ducharme J, Bartolomeos K. Emergency medicine development in Ethiopia: challenges, progress and possibilities. Afr J Emerg Med. 2013;3:3-9.

38. Ensor T, Cooper S. Overcoming barriers to health service access: influencing the demand side. Health Policy Plan. 2004;19(2):69-79.

39. Cham M, Vangen S, Sundby J. Maternal deaths in rural Gambia. Global Public Health. 2007;2(4):359-72.

40. King R: Collaboration with traditional healers in HIV/AIDS prevention and care in sub-Saharan Africa: a literature review. 2000,

41. Sundari T. The untold story: how the health care systems in developing countries contribute to maternal mortality. Int J Health Serv. 1992;22(3):513-28.

42. Pascasie K, Mtshali NG. A descriptive analysis of emergency department overcrowding in a selected hospital in Kigali, Rwanda. Afr J Emerg Med. 2014:4(4):178-83.

43. Hoot NR, Aronsky D. Systematic review of emergency department crowding: causes, effects, and solutions. Ann Emerg Med. 2008;52(2):126-36. el

44. Anest T, de Ramirez SS, Balhara KS, Hodkinson P, Wallis L, Hansoti B. Defining and improving the role of emergency medical services in cape town, South Africa. Emerg Med J. 2016;33(8):557-61. https://doi.org/10.1136/ emermed-2015-205177.

45. World Health Organization. Global status report on road safety: time for action. Geneva: World Health Organization; 2009.

46. Mayron R, Long RS, Ruiz E. The 911 emergency telephone number: impact on emergency medical systems access in a metropolitan area. Am J Emerg Med. 1984;2(6):491-3.

47. Arreola-Risa C, Mock CN, Lojero-Wheatly L, de la Cruz O, Garcia C, CanavatiAyub F, Jurkovich GJ. Low-cost improvements in prehospital trauma care in a Latin American city. J Trauma Acute Care Surg. 2000;48(1):119.

48. Carney CJ. Prehospital care-a UK perspective. Br Med Bull. 1999;55(4):757-66.

49. Mock C, Arreola-Risa C, Quansah R. Strengthening care for injured persons in less developed countries: a case study of Ghana and Mexico. Inj Control Safe Promot. 2003;10(1-2):45-51

50. Mock CN, Tiska M, Adu-Ampofo M, Boakye G. Improvements in prehospital trauma care in an African country with no formal emergency medical services. J Trauma Acute Care Surg. 2002:53(1):90-7.

51. Husum H, Gilbert M, Wisborg T. Training pre-hospital trauma care in low-income countries: the 'Village University' experience. Med Teach. 2009;2003:142-148.

52. Kumar S, Chaudhary S, Kumar A, Agarwal AK, Misra MC. Trauma care - a participant observer study of trauma centers at Delhi, Lucknow and Mumbai. Indian J Surg. 2009;71:133-41.

Ready to submit your research? Choose BMC and benefit from

- fast, convenient online submission

- thorough peer review by experienced researchers in your field

- rapid publication on acceptance

- support for research data, including large and complex data types

- gold Open Access which fosters wider collaboration and increased citations

- maximum visibility for your research: over $100 \mathrm{M}$ website views per year

At BMC, research is always in progress.

Learn more biomedcentral.com/submissions 